\title{
Electron diffraction studies of laser-pumped molecules. I. Characterization of system and analysis of data
}

\author{
L. S. Bartell, M. A. Kacner, and S. R. Goates \\ Department of Chemistry, University of Michigan, Ann Arbor, Michigan 48109 \\ (Received 27 April 1981; accepted 12 June 1981)

\begin{abstract}
The feasibility of investigating the vibrational excitation of laser-pumped molecules in a supersonic jet by electron diffraction is investigated. Studies of $\mathrm{SF}_{6}$ confirm that diffraction patterns can be measured, reproducibly, to a part per thousand of the total intensity, or better, and hence can be analyzed to yield useful information about laser-induced changes in intramolecular motions, provided adequate account is taken of certain systematic errors. Procedures for discriminating between bona fide and spurious signals are outlined. Methods are described for characterizing the gas flow and density profile in the microjets issuing from a small tubular nozzle, and for measuring the distribution of radiant energy in the focused infrared beam used to irradiate the gas jet.
\end{abstract}

\section{INTRODUCTION}

The vibrational excitation of small molecules by lasers has been actively investigated in the last few years with particular attention paid to sulfur hexafluoride. ${ }^{1-4}$ At the outset of the present investigation it appeared that several of the problems resisting analysis by conventional spectroscopic approaches might be amenable to electron diffraction methods. Electron diffraction, by measuring amplitudes of molecular vibrations, should in principle be capable of following the absorption and redistribution of radiant energy in gas-phase molecules. Possible advantages and limitations are discussed in paper $\mathrm{II}^{5}$ of this series. In order to carry out a fruitful analysis by electron diffraction, however, it was clear that new procedures had to be developed in order to attain the requisite precision. As this research progressed it was found that the sensitivity could indeed be augmented considerably over that customarily achieved. Because the analyses adopted introduce unconventional procedures, they warrant a fairly detailed accounting. Moreover, the interpretation of results depends critically upon several experimental parameters. Therefore an outline of how the system was characterized is presented.

\section{EXPERIMENTAL}

\section{A. Apparatus}

The experimental arrangement is shown schematically in Fig. 1. Acw infrared beam from a tunable Advanced Kinetics $50 \mathrm{WCO}_{2}$ laser is directed through a zinc sele nide lens ( 5 in. focal length) by reflection from an adjustable mirror, and thereby focused onto a microjet of sulfur hexafluoride issuing from a fine nozzle. Pumped molecules are probed downstream by a $40 \mathrm{keV}$ electron beam after a preset (time-of-flight) interval. In order to facilitate the precise alignment of the focused infrared beam, the $\mathrm{ZnSe}$ focusing element is also put to use as the objective lens of an optical microscope (Fig. 2) affording a parallax-free visual image of the focused spot and gas nozzle. Inspection of the laser spot can be carried out with the aid of a suitable carbon microscreen capable of being swung into place in back of the nozzle. During alignment a chopper reduces the brightness of the image to comfortable limits. Adjustments of the position of the focused spot can be made with micrometer screws on the $45^{\circ}$ mirror, and the location of the spot with respect to the nozzle can be measured to better than a tenth of a millimeter. Sodium chloride beam splitters are moved into the inf rared beam as desired, to permit sampling of the output wattage and wavelength.

A description of the electron diffraction apparatus has been published elsewhere. ${ }^{6}$ In the present experiments the gas nozzle was a hypodermic needle $0.12 \mathrm{~mm}$ in diameter with its stainless steel microtube cut off at the nickel-plated brass hub in order to reduce radiant heating of the tip. Such heating can be enormous if the laser beam grazes the nozzle ${ }^{7}$ and, in the present work, it turned out to contribute the largest source of error. The tubular nozzle, $4 \mathrm{~mm}$ long after cutting, was placed 21 $\mathrm{cm}$ above the photographic plates recording the electron diffraction patterns.

\section{B. Gas jet density}

Computations of average laser fluence experienced by molecules flowing from the nozzle and estimates of collision numbers and the temperature distribution in the

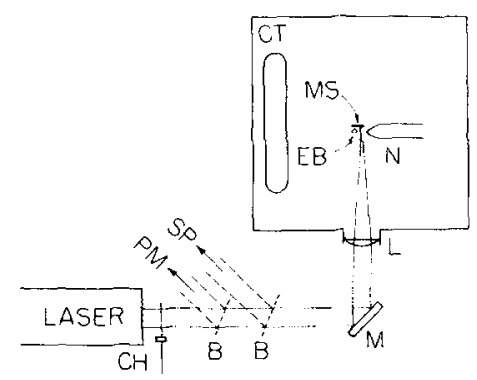

FIG. 1. Schematic diagram of experimental arrangement. Adjustable mirror $M$ directs laser beam through $\mathrm{ZnSe}$ lens $\mathrm{L}$ into electron diffraction apparatus. The infrared beam is focused upon a microjet issuing from nozzle N. Microscreen MS can be brought into position to provide a visual image of the position of the focused infrared spot relative to the nozzle tip. The gas jet is probed by electron beam EB (perpendicular to plane of figure) after irradiation, then condensed on cold trap CT.

Beamsplitters $B$ can be turned into place to direct a known portion of the beam into a spectrometer SP and power meter PM. Chopper $\mathrm{CH}$ attenuates the laser output during alignment procedures. 


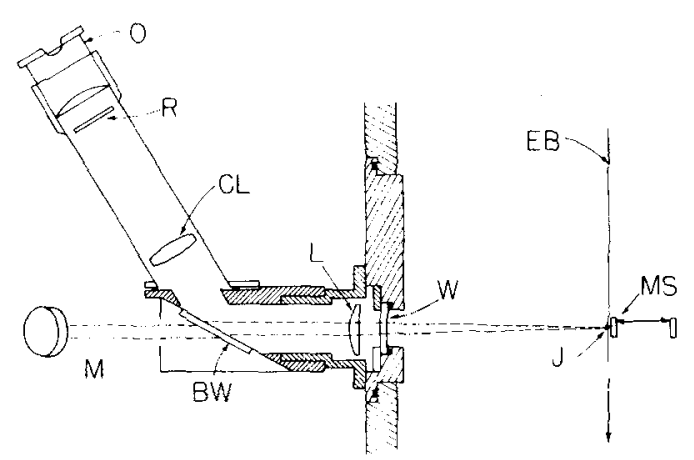

FIG. 2. Collimator-microscope system for focusing infrared beam and viewing it along the same axis. Mirror M (see Fig. 1) directs laser beam through Brewster window BW, coated lens $\mathrm{L}$, and coated vacuum window $\mathrm{W}$ onto gas jet $\mathrm{J}$ (perpendicular to plane of figurel. Carbon microscreen MS can be positioned at laser focus to render it visible. Visible light emitted from screen MS is focused by lens $\mathrm{L}$, reflected efficiently by BW along the optic axis of the microscope, and transmitted through correction lens CL. An image of the laser focus and nozzle tip at reticle $R$ is viewed through ocular $O$. Electron beam EB probes gas jet $\mathrm{J}$ downstream of laser focus. All infrared transmission opties are $\mathrm{ZnSe}$.

supersonically cooled gas jet require information about gas throughputs and density profiles. Throughputs were computed from the rate of pressure change in a sample reservoir of known volume as the gas flowed through the nozzle into the evacuated electron diffraction specimen chamber.

Density profiles across the gas jet were determined by translating the gas nozzle, thereby moving the gas jet through a fixed electron beam, and measuring a fixed fraction of the scattered electron current with a picoammeter. From measurements of the electron current

$$
I_{e}(y) \approx K \int_{-\infty}^{\infty} \rho(y, z) d z
$$

scattered by gas distributed along the electron beam direction $z$ as a function of lateral displacement $y$ of the nozzle (Fig. 3), it is possible to deduce $\rho(y, z)$ for the gas jet if the flow is assumed to have axial symmetry. In probing $\rho(y, z)$ the diameter of the electron beam was reduced to a value small compared with the diameter of the gas jet to simplify corrections for the finite breadth of the electron beam.

\section{Intensity profile at laser focus}

Infrared power densities were measured both for the unfocused and focused laser beams. This was done by translating a straightedged beam stop across the beam by means of a micrometer screw. Measurements of the laser power on the lee side of the beam stop as a function of the block position can be interpreted in terms of the power density $W(r)$ of the laser beam, if it is assumed that the beam is circularly symmetric. A beam stop capable of withstanding the power densities encountered was constructed from a thick aluminum block, clad on the irradiated side with a polished stainless steel strip. Even with a lens of comparatively long focal length (5 in.) the focused spot at full output is sufficiently intense to damage steel surfaces quickly if they are tarnished or dirty. Therefore, the reflector block was kept scrupulously clean and the laser beam was greatly attenuated with a chopper of small transmission. The chopping blade's twin transmission slots were machined to be substantially larger than the laser beam in order to minimize diffraction broadening of the focused beam by the chopper.

Laser outputs corresponding to $\mathrm{TEM}_{00}$ modes are supposed to be very nearly Gaussian in power density. ${ }^{8}$ Therefore, the distribution across the focused spot from a $\mathrm{TEM}_{00}$ mode would also be expected to be Gaussian. Because the amplitude distribution at the focus of a thin lens, focal length $f$, is the Fourier transform of the amplitude transmitted by the lens, an unfocused amplitude

$$
\left[W_{u}(r)\right]^{1 / 2}=\left[W_{M} \exp \left(-r^{2} / 2 \sigma_{u}^{2}\right)\right]^{1 / 2}
$$

of a coherent wave incident upon the lens should focus to an amplitude

$$
\left[W_{f}(r)\right]^{1 / 2}=\left[W_{m} \exp \left(-r^{2} / 2 \sigma_{f}^{2}\right)\right]^{1 / 2}
$$

on the focal plane, where

$$
\sigma_{f}=f \lambda / 4 \pi \sigma_{u} .
$$

If the distribution is given by Eq. (2) or (3), the power $\dot{E}_{\mathrm{tr}}$ not blocked by the steel-clad block translated in the $x$ direction should vary with $x$ as

$$
\dot{E}_{\text {tr }}(x)=\frac{1}{2} \dot{E}\left[1+\operatorname{erf}\left(x / 2^{1 / 2} \sigma\right)\right] .
$$

From a knowledge of the laser power $\dot{E}$ and the standard deviation $\sigma$ of a Gaussian distribution it is elementary to calculate the power density

$$
W_{\max }=\dot{E} / 2 \pi \sigma^{2}
$$

at the center of the laser beam (focused or unfocused).

\section{Electron diffraction studies}

A precise specification of all experimental conditions will be deferred until paper II. ${ }^{5}$ Suffice it to mention here that over 200 diffraction plates were taken at $\mathbf{S F}_{6}$ sample pressures ranging from 50 to 600 Torr, and at various laser powers and wavelengths. Included were 41 plates, one from each set of five diffraction exposures, taken without laser irradiation of the sample. Here-

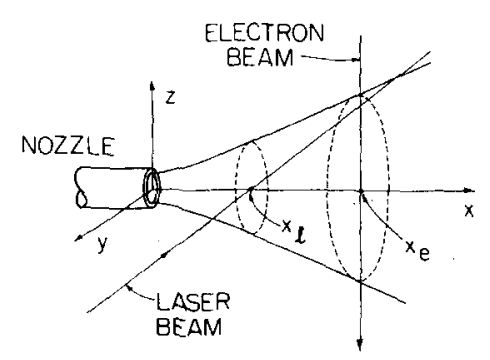

FIG. 3. Coordinate system pertaining to Eqs. (1) and (8)-(12). Origin at center of nozzle tip. The focused laser beam intersecting the gas jet axis at $x_{l}$ is virtually cylindrical in its path through the jet. 
after these plates will be referred to as "control plates." Unfortunately, the full breadth of the fringe of the focused laser beam was not measured or fully appreciated until after the above runs were made. Later tests with a thermocouple embedded in the nozzle indicated that radiant heating of $5^{\circ}$ to $25^{\circ}$ was possible during exposures. Analyses described in the following verify, however, that nozzle heating effects did not interfere critically with our observations of direct laser pumping.

The extremely large number of plates taken (as compared with typically fewer than a half dozen at a given camera geometry in standard studies) afforded a rare and valuable opportunity to carry out diagnoses and tests. These were crucial in the formulation of a revised procedure for analysis of the diffraction data. Several aspects of this analysis are discussed in the next section.

\section{ANALYSIS OF DATA}

\section{A. Treatment of diffraction data}

It will be assumed that interested readers are already familiar with standard procedures and notation in the field. ${ }^{6,9}$ Before describing deviations of our approach from conventional approaches it is instructive to outline both the special problems encountered when studying pumped molecules and the characteristic features of the data that suggested the procedural modifications we introduced.

Of primary importance is the need to increase by almost an order of magnitude the precision of measuring amplitudes of vibration. This must be accomplished despite the severe and uncharacteristic limitation in the range of scattering angle monitored. A curtailed range was a compromise dictated by the difficulty of the experiment and the great number of variables to be explored which made it impractical to use more than a single camera geometry. Moreover, the "long camera" geometry we adopted is not the optimum geometry for studying amplitudes with delicacy. An additional adverse factor was the observation of large nonrandom residuals in the comparison between experiment and standard diffraction theory in the range studied. In fact, in a prior conventional study of $\mathrm{SF}_{6}{ }^{10}$ these residuals had been considered to be so intolerably large that considerable effort was spent in analyzing the sample for contaminants; contaminants were eventually ruled out as the culprit, however. Corresponding data taken for the similar molecule $\mathrm{SF}_{5} \mathrm{Cl}$ at about the same time ${ }^{11} \mathrm{re}-$ vealed comparable residuals-residuals considered so threatening to the derivation of accurate structure parameters that the long camera data were simply discarded. Residuals of the magnitude encountered, when incorporated into conventional (rather blind) error analyses, imply uncertainties in amplitudes far too great to make a study of laser pumping feasible.

Two factors changing the complexion of this most unpromising situation are as follows. Experimentally, we found from our 41 control plates that our residuals exhibit extraordinary reproducibility. Theoretically, we found reasons why conventional diffraction theory is

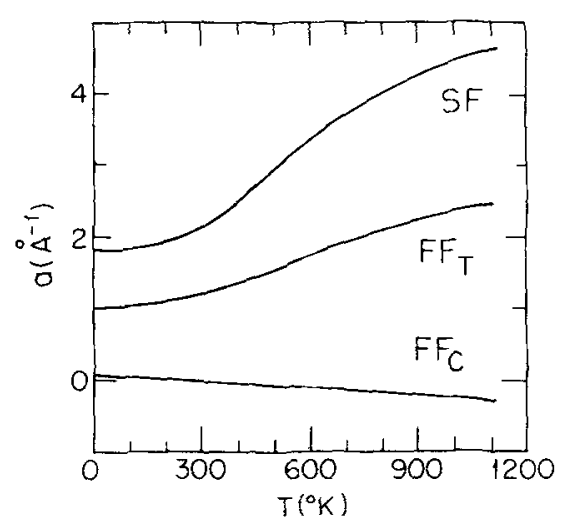

FIG. 4. Temperature dependence of $a$, the effective Morse asymmetry parameter for the three types of atom pairs in $S_{6}$.

deficient in its representation of small angle intensities. ${ }^{12,13}$ Both factors suggest that the absolute accuracy of derived amplitudes of vibration is significantly imperfect, and is likely to remain imperfect despite recent improvements introduced into the theory. What makes this irrelevant to the present study is that our requirements are only for precision, not accuracy, since it is only changes induced in molecules by irradiation that are of consequence. Those changes turn out to be insensitive to the systematic defects in theory (and experiment) that give rise to the residuals in the first place.

Before ascribing the systematic residuals principally to oversimplifications in theory, we made a painstaking search for possible flaws in our apparatus and treatment, examining with special care our rotating-sector calibration, our tables of atomic scattering factors, the mechanics of our computer programs, and our computer inputs. Residuals were significantly decreased by incorporating corrections for intramolecular multiple ("dynamic") scattering according to expressions developed independently in this laboratory and by Kohl and Arvedson. ${ }^{13}$ It is plausible that much of the remainder of the residuals can be attributed to deviations of electron densities from those implied by the independentatom-model incorporated into scattering factors as discussed by Kohl and Bartell, ${ }^{14}$ and Fink et al. ${ }^{15}$

Whatever the origin of the residuals, their reproducibility made it reasonable to carry out refinements of data by the following procedure. Corrections for intramolecular multiple scattering were applied to the 41 control plates using the Kohl-Arvedson numerical results. ${ }^{13}$ Refinements for each control plate were then carried out with the imposition of molecular geometric self-consistency (incorporating shrinkage corrections of $0.00063 \AA$ cis and $0.00262 \AA$ trans) and room temperature asymmetry values ${ }^{16}$ (Fig. 4). Background functions (quartic polynomial plus exponential term) were refined simultaneously with molecular parameters. ${ }^{17}$ Residuals $\Delta M_{r}(s)$ so obtained for each plate were then averaged to give a $\Delta \bar{M}_{r}(s)$ function to be incorporated, with modification, in the analysis of the laser-irradiated plates. When the individual control plates were each corrected by $\Delta \bar{M}_{r}(s)$ and refined once more, the surviving residuals of the 
individual control plates dropped by a factor of 5 and lost their conspicuously systematic pattern.

Diffraction intensities for laser-irradiated samples were initially refined as in the initial control plate refinements with room temperature asymmetry values and multiple scattering corrections without introducing $\Delta M_{r}(s)$ corrections, but internuclear distances were refined individually without the imposition of geometric constraints. These refinements led to estimates of the vibrational temperatures and permitted updating the asymmetry values ${ }^{16} a(T)$. Mean residual corrections $\Delta \bar{M}_{r}(s)$ from the control plate were then introduced and crudely corrected, as were also the multiple scattering corrections, for effects of temperature by multiplication by the factor $\exp \left[-\left(l_{T}^{2}-l_{298}^{2}\right) s^{2} / 2\right]$, where $l$ represents the $S-F$ mean amplitude. In future studies less primitive temperature corrections would be desirable.

\section{B. Diagnosis of optical vs thermal excitation}

As mentioned previously, the present experimental arrangement gave marginal protection against heating the nozzle tip by the fringe of the focused laser spot. One way to discriminate between vibrational excitation of the sample acquired by its passage through a heated nozzle and excitation induced by direct infrared pumping is to observe the excitation as a function of infrared frequency. Thermal excitation should be independent of small changes in wavelength but pumping should parallel the absorption spectrum. Results of such an examination are given in paper II. ${ }^{5}$ A direct test at a single laser frequency is also possible as outlined in the following.

Initial experiments had been carried out with an unprotected nozzle and led to a considerable thermal excitation of $\mathrm{SF}_{6} \cdot{ }^{7}$ It was recognized at once that the excitation was brought about primarily by heating of the gas as it flowed through a hot nozzle, however, because the diffraction plates were characteristically less heavily exposed, the greater the excitation. Such a diminution of exposure could only have resulted from a smaller concentration of gas molecules in the region probed by the electron beam. Laser pumping could not have depleted the gas because too few collisions take place in our experiment for $V-T$ relaxation. ${ }^{18}$ A hot nozzle diminishes gas concentration downstream in two ways. First, gas viscosity increases as the temperature increases, thereby cutting down the flow rate. Secondly, the translationally excited molecules spend less time in their flight across the electron beam, A study with a heated nozzle yielded a linear plot of $\log \left(E / E_{c}\right)$ vs $\log T_{\text {vibr }}$, where $E$ and $E_{c}$ are photographic exposures observed for thermally excited samples and control (unheated) samples, respectively, each taken at the same stagnation pressure and normalized to the same exposure time and electron beam current. ${ }^{19}$ Therefore a plot of $\log \left(E / E_{c}\right)$ against $\log T_{\text {vbr }}$ reveals the origin of the excitation. Thermal excitation induced by a hot nozzle tip is recognized by a characteristic slope ${ }^{19}$ of about $-\frac{2}{3}$. Laser pumping should yield a slope of nearly zero.

\section{RESULTS}

\section{A. Gas jet density and flow}

Varying the stagnation pressure in the $\mathrm{SF}_{6}$ sample bulb from 50 to 600 Torr varied the throughput $Q$ from about 0.01 to 0.4 Torr $\ell \mathrm{s}^{-1}$. Gas pressures $P_{N}$ at the exit of the nozzle were estimated by assuming that the flow through the metallic nozzle was approximately isothermal, following the Langhaar-Worden flow theory ${ }^{20}$ at pressures low enough to avoid choked flow at the tip. In the case of choked flow at higher pressure the jet exit velocity $v_{N}$ was taken as sonic (i.e., at a Mach number of $M \approx 1$ ) at maximum. A knowledge of $Q, \bar{v}_{N}$, and nozzle diameter $D$ established the exit pressure

$$
P_{N}=4 Q / \pi D^{2} \vec{v}_{N}
$$

to be in the range of 8-200 Torr in our experiments. As the gas expands adiabatically into the vacuum chamber, its pressure drops very rapidly. In the case of flows from thin plate orifices, the drop in concentration and temperature can be calculated from the relations of Ashkenas and Sherman ${ }^{21}$ who found that the gas density several nozzle diameters away from the orifice is given accurately by

$$
\rho(y, z)=\rho_{\max } \cos ^{2} \theta \cos ^{2}(\pi \theta / 2 \phi)
$$

in the plane at a distance $x$ from the orifice, where $\theta$ is the angle of apparent divergence of the gas from a point $x_{0}$, and where $x_{0}$ and the angular divergence parameter $\phi$ are tabulated functions of $\gamma=C_{p} / C_{v}$ for the gas.

For flow from our tubular nozzles it could not be assumed that Eq. (8) applied. Therefore, scattered electron currents $I_{e}(y)$ of $\mathrm{Eq}$. (1) were determined for several pressures and two nozzle-to-electron beam distances. From our finding that

$$
I_{e}(y) \approx I_{\max }\left[1+b(y / x)^{2}\right]^{-3 / 2}
$$

we infer that the gas density is well represented by a "second-order Witch of Agnesi",22

$$
\rho(R)=\rho_{\max }\left[1+b(R / x)^{2}\right]^{-2},
$$

with $R^{2}=y^{2}+z^{2}$, where $b$ is a parameter characterizing the diffuseness of the gas jet. Now, Eq. (10) is similar in functional form to the Ashkenas and Sherman density ${ }^{21}$ $[\mathrm{Eq} .(8)]$ in the region of appreciable gas concentration. In fact, if $\phi=\pi / 2$, a value in the midrange of tabulated values, Eqs. (8) and (10) are identical. Jets issuing from our tubular nozzles were significantly narrower, however, than jets produced by thin plate orifices according to the theory of Ashkenas and Sherman. Measurements for $\mathrm{SF}_{b}$ yielded a value of parameter $b$ of about 2. This can be shown ${ }^{23}$ to correspond to $\phi \approx 1.15$, whereas $\phi$ for a thin-plate orifice would be much larger, or about 1.9 at the effective $\gamma$ value for our microjet $(\gamma \sim 1.25-1.3) .{ }^{23}$

A discussion of the relation between parameters $\phi$ and $b$, and their role in the computation of jet velocities and temperatures will be presented in paper III. ${ }^{23}$

\section{B. Intensity profile at laser focus}

The unfocused laser beam at about $40 \mathrm{~W}$ was observed to be approximately Gaussian with $\sigma_{u} \approx 0.17 \mathrm{~cm}$, and the 
focused spot was very well represented by a Gaussian function with $\sigma_{f} \approx 0.015 \mathrm{~cm}$. Because $\sigma_{f}$ is twice the breadth calculated according to Eq. (4), it appears that the contributions to various areas of $W_{u}(r)$ are not all mutually coherent. At the center of the focused spot the power density $[\mathrm{Eq} .(6)]$ is about $25 \mathrm{~kW} / \mathrm{cm}^{2}$. At the nozzle tip, the power density has fallen to $4 \mathrm{~kW} / \mathrm{cm}^{2}$ or less when the laser beam is at its usual position, 0.3 to $0.35 \mathrm{~mm}$ beyond the nozzle. The breadth of the laser beam does not increase very rapidly inside or outside of the exact focal point because of the comparatively long focal length of the lens. Within the microjet of the gas sample, the focused laser beam is nearly cylindrical, not conical, because of diffraction.

\section{Mean fluence}

Molecules in the gas jet are subjected to a distribution of energy density (fluence), with molecules far off the axis of the jet experiencing a much lower integrated fluence than those directed through the center of the focused laser beam. If the spatial distributions characterizing the gas jet and laser beam are known, it is straightforward to calculate the average fluence $\left\langle\Phi_{e}\right\rangle$ imposed upon gas molecules that are subsequently sampled by the electron beam. Taking $x, y$, and $z$ as the axes, respectively, of the gas jet, the laser beam, and the electron beam, we write for a given streamline in this jet diverging at angle $\theta$,

$$
\begin{aligned}
d \Phi / d u & =W_{f}(x, z) d t / d x \\
& =W_{f}(x, z) /\left(v_{j} \cos \theta\right)
\end{aligned}
$$

for a laser beam focused at $x_{L}$. When the increments $d \Phi$ are averaged over the density in the $y=0$ plane sampled by the electron beam and summed over the molecular paths, the result is

$$
\left\langle\Phi_{e}\right\rangle=\int_{0}^{x_{e}}\left[\frac{\int_{-\infty}^{\infty} \rho(y=0, x, z)(d \Phi / d u) d z}{\int_{-\infty}^{\infty} \rho(y=0, x, z) d z}\right] d x,
$$

where $x_{e}$ is the distance of the electron beam from the nozzle tip. If the jet density $\rho(x, y, z)$ of Eq. (10) and power density $W_{f}(x, z)$ of Eq. (3) are inserted into Eq. (12) along with the typical values $b \approx 2, \dot{E} \approx 40 \mathrm{~W}, \sigma_{f}$ $\approx 0.015 \mathrm{~cm}, v_{f} \approx 3.4 \times 10^{4} \mathrm{~cm} / \mathrm{s}, x_{L} \approx 0.03 \mathrm{~cm}$, and $x_{e}$

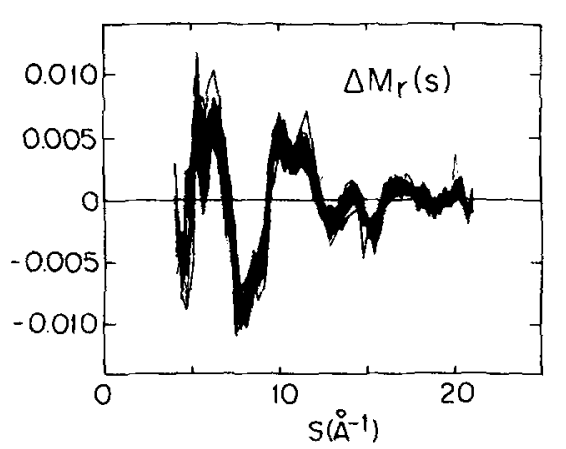

FIG. 5. Superposition of the residuals $\Delta M_{r}(s)$ of the 41 control plates after correcting for multiple scattering. Ordinate $\Delta M_{\text {, }}$ is dimensionless and represents the fractional correction of the total intensity.

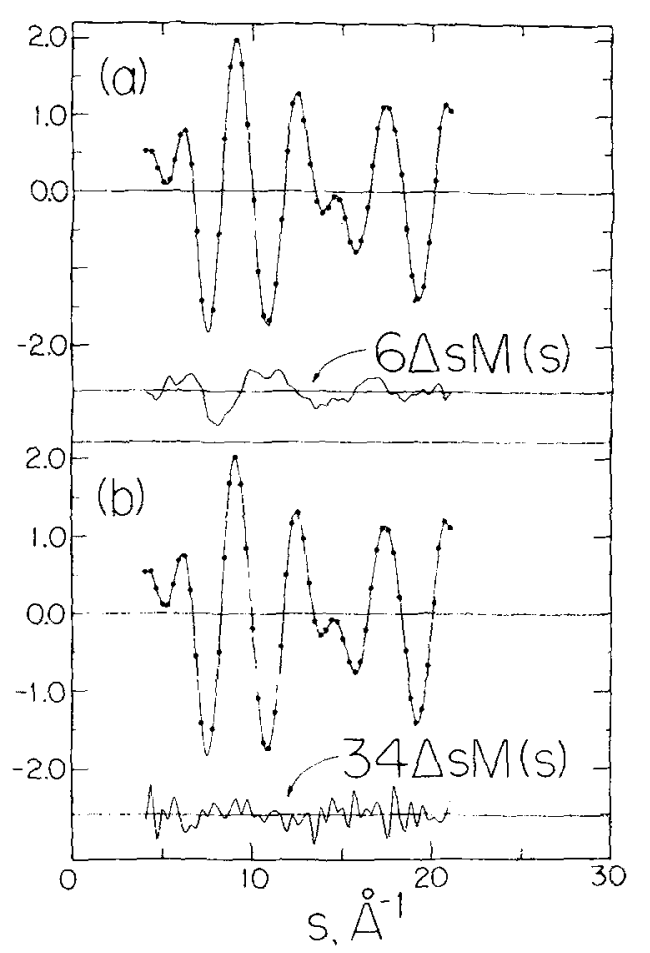

FIG. 6. Experimental (dots) and calculated molecular intensity functions $s M(s)$ for $\mathrm{SF}_{6}$, and greatly magnified residuals $\Delta s M(s)=s M_{\text {expt }}-s M_{\text {calc }}$. Illustrated are two different refinements of the same data for a characteristic plate. (a) Before correction for mean residual, showing substantial and systematic discrepancies; (b) after correction, showing small and random discrepancies.

$\approx 0.064 \mathrm{~cm}$, numerical integration yields $\left\langle\Phi_{e}\right\rangle \approx 0.021$ $\mathrm{J} / \mathrm{cm}^{2}$.

\section{Electron diffraction analysis}

Illustrating the reproducibility of the residuals in the control plates is Fig. 5, showing a superposition of the residuals $\Delta M_{r}(s)$ of all 41 control plates. How the residuals compare with the molecular interference terms themselves, and how they are reduced in a characteristic plate when the average residual $\Delta \bar{M}_{r}(s)$ is used to correct the individual plate is depicted in Fig. 6. Not only is the misfit considerably lessened by the $\Delta \bar{M}_{r}(s)$ correction but residuals lose their high point-to-point correlations. Before correction the $\Delta M_{r}(s)$ curves exhibit such a large correlation between nearby $s$ values that the effective number of independent observations appears to be small. After correction the residuals can be used quite reliably in the calculation of an error matrix for derived parameters by the standard random error theory. ${ }^{24}$ To be sure, the elements of the error matrix are no longer satisfactory for the deduction of absolute errors in the derived parameters; the large systematic oscillations in $\Delta \bar{M}_{r}(s)$ can distort derived parameters by perhaps several thousandths of an Angstrom unit. What is more important is that the error matrix now yields the more crucial information about how sensitive the data are to changes in intermolecular distances and amplitudes of vibration. And the sensitivity is found to be of the order of ten thousandiths of an angstrom unit, an or- 
der of magnitude higher than the accuracy commonly obtained in conventional studies.

\section{CONCLUSION}

A revised procedure for analysis of electron diffraction patterns has been developed. When tested with a large body of new diffraction data, it demonstrates that amplitudes of vibration can be derived with a precision high enough to be useful in investigations of vibrational excitation in laser-pumped gas molecules. An experimental arrangement for such laser studies has been devised. Procedures for characterizing properties of the supersonic gas jet and focused laser beam are described. These properties are needed for the interpretation of vibrational excitation observed when a microjet of sulfur hexafluoride streams through the focus of an infrared laser. Observations are described in the following paper.

\section{ACKNOWLEDGMENTS}

This research was supported by the National Science Foundation under grant number CHE -7926480. We thank Mr. William Vance for assistance in measuring the pow er density of the laser beam. A generous allotment of computer time was donated by the University of Michigan Computing Center.

${ }^{1}$ J. P. Aldridge, J. M. Birely, C. D. Cantrell, and D. C. Cartwright, in Physics of Quantum Electronics, edited by S, F. Jacobs, M. Sargent, and C. T. Walker (Addison-Wesley, Reading, Mass., 1976), Vol. 4.

${ }^{2}$ R. V. Ambartzumiam and V. S. Letokhov, Acc. Chem. Res. 10, 61 (1977); N. Bloembergen and E. Yablonovitch, Phys. Today 31, (No. 5), 23 (1978).

${ }^{3}$ J. I. Steinfeld, I. Burak, D. G. Sutton, and A. V. Nowak, J. Chem. Phys. 52, 5421 (1970); D. S. Frankel, ibid. 65, 1696 (1976); T. F. Deutsch, Opt. Lett. 1, 25 (1977); D. Mann and M. Rothchild, ibid. 1, 28 (1977).
${ }^{4}$ See, for example, P. A. Schulz, Aa. S. Sudbe, D. J. Krajnovich, H. S. Kwok, Y. R. Sen, and Y. T. Lee, Annu. Rev. Phys. Chem. 30, 379 (1979).

${ }^{5}$ L. S. Bartell, M. A. Kacner, and S. R. Goates, J. Chem. Phys. 75, 2736 (1981).

${ }^{6}$ L. S. Bartell, in Physical Methods in Chemistry, edited by A. Weissberger and B. W. Rossiter (Interscience, New York, 1973), 4th edition.

${ }^{7}$ L. S. Bartell, S. K. Doun, and S. R. Goates, J. Chem. Phys. 70, 4585 (1979).

${ }^{8}$ M. Sargent, M. O. Scully, and W. E. Lamb, Jr., Laser Physics (Addison-Wesley, Reading, Mass., 1974), p. 368.

${ }^{9}$ See, for example, L. S. Bartell, K. Kuchitsu, and H. M. Seip, Acta Crystallogr. Sect. A 32, 1013 (1976).

${ }^{10}$ L. S. Bartell and S. K. Doun, J. Mol. Struct. 43, 245 (1978).

${ }^{11}$ C. J. Marsden and L. S. Bartell, Inorg. Chem. 15, 3004 (1976).

${ }^{12}$ B. R. Miller and L. S. Bartell, J. Chem. Phys. 72, 800 (1980).

${ }^{13}$ D. A. Kohl and M. Arvedson, J. Chem. Phys. 72, 1922 (1980).

${ }^{14}$ D. A. Kohl and L. S. Bartell, J. Chem. Phys. 51, 2891, 2896 (1969).

${ }^{15}$ See, for example, M. Fink, P. G. Moore, and D. Gregory, J. Chem. Phys. 71, 5227 (1979).

${ }^{16}$ L. S. Bartell, J. Mol. Struct. 63, 259 (1980).

${ }^{17}$ L. S. Bartell, D. A. Kohl, B. L. Carroll, and R. M. Gavin, Jr., J. Chem. Phys. 42, 3079 (1965).

${ }^{18}$ C. L. O'Connor, J. Acoust. Soc. Am. 26, 361 (1954); T. L. Cottnell and J. C. McCoubrey, Molecular Energy Transfer in Gases (Buttersworth, London, 1961).

${ }^{19} \mathrm{~S}$. K. Doun, thesis (University of Michigan, 1979).

${ }^{20}$ D. G. Worden, in Scientific Foundations of Vacuum Technique, edited by J. M. Lafferty (Wiley, New York, 1962), pp. 84-85.

${ }^{21}$ M. Ashkenas and F. S. Sherman, Rarified Gas Dynamics, Fourth Symposium, edited by J. M. deLeeuw (Academic, New York, 1966), Vol. II, p. 84.

${ }^{22}$ For a generalization of the term "Witch of Agnesi" to encompass $n$th order functions, see H. Hauptman and J. Karle, Phys. Rev. 77, 491 (1950).

${ }^{23}$ L. S. Bartell, M. A. Kacner, i. R. Goates (unpublished research).

${ }^{24}$ See, for example, L. S. Bartell and I M. G. Anashkin, J. Mol. Struct. 17, 193 (1973). 